\title{
BMJ Open Validity of self-reported age at menarche in computer-assisted interview among Chinese schoolgirls: a cross-sectional study
}

\author{
Yanyan Mao, ${ }^{1,2}$ Qiguo Lian, ${ }^{1,2}$ Xiayun Zuo, ${ }^{2}$ Yan Zhang, ${ }^{1,2}$ Shan Luo, ${ }^{3}$ \\ Shucheng Zhang, ${ }^{4}$ Xiaowen $\mathrm{Tu}^{2}{ }^{2}$ Chaohua Lou, ${ }^{2}$ Weijin Zhou ${ }^{2}$
}

To cite: Mao Y, Lian Q, Zuo X, et al. Validity of self-reported age at menarche in computerassisted interview among Chinese schoolgirls: a crosssectional study. BMJ Open 2018:8:e016799. doi:10.1136/ bmjopen-2017-016799

- Prepublication history and additional material for this paper are available online. To view these files, please visit the journal online (http://dx.doi. org/10.1136/bmjopen-2017016799).

Received 10 March 2017 Revised 20 September 2017 Accepted 20 November 2017

Check for updates

${ }^{1}$ School of Public Health, Fudan University, Shanghai, China

${ }^{2}$ Key Laboratory of Reproduction Regulation of NPFPC, SIPPR, IRD, Fudan University, Shanghai, China

${ }^{3}$ West China Second University Hospital, Sichuan University,

Chengdu, China

${ }^{4}$ Reproductive Physiology Laboratory, National Research Institute for Family Planning, Beijing, China

Correspondence to

Professor Weijin Zhou;

zhouweijin@sippr.org.cn

\section{ABSTRACT}

Objective The attitudes of girls regarding menarche vary according to their cultural backgrounds. Asian girls may hesitate to discuss menarche. Computer-assisted selfadministered interviewing (CASI) is considered a valid and effective tool for investigating the timing of menarche; however, the validity of self-reported menarche data from CASI in Chinese culture is currently unknown. We aimed to validate the status and age of menarche attainment by comparing CASI with face-to-face interviewing (FFI).

Methods Based on a cross-sectional study, we collected information regarding the status and age of menarche attainment using CASI and FFI among Chinese schoolgirls. We explored the tools of standard epidemiological indices, including sensitivity, specificity, predictive values and accuracy to examine the capacity of CASI for correctly classifying the status of menarche. Both Pearson and Spearman correlations were calculated for the correlation of age at menarche using CASI with FFI. A Bland-Altman plot was drawn to measure the agreement between the two interview techniques.

Results In this study, CASI and FFI were conducted in 3478 schoolgirls with an average age (SD) of 14.3 years (2.46). Menarche attainment was reported in 2496 (71.2\%) and 2538 (73.0\%) girls using CASI and FFI, respectively. Compared with FFI, the sensitivity, specificity, positive predictive value (PPV), negative predictive value and the accuracy of CASI were $0.97,0.80,0.96$ and 0.92 , respectively. The correlation of age at menarche between CASI and FFI was 0.728 . Approximately $>95 \%$ and $76.3 \%$ of the difference in the age at menarche was within 12 months and 3 months, respectively, between the two interview methods. Among primary schoolgirls in grade 5 , the sensitivity, specificity, PPV, accuracy and the correlation (0.335) of CASI was lower than those in other subgroups.

Conclusions Overall, the timing of menarche investigated using CASI was valid among all Chinese schoolgirls, except for those in grade 5 or lower.

\section{INTRODUCTION}

Menarche, one of the milestones in pubertal development, is regarded as a unique maturation marker of transition from girls' childhood to adulthood and is considered

\section{Strengths and limitations of this study}

- We validated the self-reported age at menarche using computer-assisted self-administered interviewing (CASI), in comparison with face-toface interviewing (FFI), in a large population of 3478 schoolgirls in six provinces in China nationwide.

- The correlations in subgroups according to locations, grades, awareness in reproductive health and breast tanner stages were determined, and the correlation of the age at menarche reported using CASI with FFI was poorer among girls in lower grades or those with poor reproductive health knowledge.

- The data collected using CASI and FFI were selfreported and not prospectively recorded in this study.

an essential public health target for sexual education. The age at menarche is related to mental disorders. ${ }^{1}$ Both early and late menarche were associated with an increased risk of vascular disease. ${ }^{2}{ }^{3}$ Therefore, a valid assessment of menarcheal age is fundamentally needed for epidemiology studies that are conducted to evaluate the impact of timing of menarche. Self-reporting of menarcheal age by face-to-face interviewing (FFI) has been commonly used and considered valid and effective for evaluating the timing of menarche by short-term recall. ${ }^{45}$

Girls' attitude to menarche has been found to vary according to their different cultural backgrounds. ${ }^{6} 7$ In Asian countries, girls are more sensitive and conservative regarding reproductive health issues. Therefore, Asian girls tend to feel ashamed when talking about menarche. Computer-assisted self-administered interviewing (CASI), in which respondents read the questions of a survey on a computer screen and then directly enter their responses into a computer, has been applied for investigating reproductive 
health to improve the quality and efficiency of self-reported data. ${ }^{8}$ In addition to cost-saving, a higher reporting of adolescent sexual behaviour, drug use and violence in surveys of risk behaviours were observed with CASI for school-aged children in comparison with FFI or paper-and-pencil self-administered interviews. ${ }^{9} 10$ This was because the CASI provides increased privacy of responses and the freedom for reporting behaviours that are widely believed to be risky or socially undesirable. ${ }^{1112}$ However, FFI for assessing adverse experiences is generally regarded as superior to self-administered interviewing because FFI allows for probing and clarification of relevant details and minimises biases related to subjective responses. ${ }^{13}$ To our knowledge, there are currently no studies conducted to examine the validity and reliability of self-reported menarche data by CASI in Chinese culture.

The objective of the present cross-sectional study was to confirm the validity of the status and age of menarche attainment among schoolgirls in China obtained using CASI in comparison with FFI.

\section{METHOD}

\section{Population and sample}

This cross-sectional study was conducted in six provinces in China from November 2012 to April 2013. A total of 4290 girls from 26 sampled schools were recruited. These girls were sampled using the multistage cluster sampling strategy. ${ }^{14}$ There were 24 girls with missing records regarding menarche and 788 girls refused to undergo the physical examination (online supplementary table S). Finally, 3478 girls were interviewed by both CASI and FFI.

Both parental permission and students' consent were obtained for all participants before the survey.

\section{Data collection}

Sawtooth Ci3, V. 2.6.16 (Sawtooth software, Inc., Utah, USA) was used to compile the questionnaire that was administered using personal computers. During CASI, girls were interviewed with two main questions regarding their menarche. The first question was 'do you have menstrual periods?'; if the answer was 'yes', they were asked 'how old were you when you started your first period?'. Additional questions were asked as necessary to probe for a more accurate date of menarche attainment (eg, 'what grade in school were you?' and 'what season was it?'). Girls were also tested for their awareness in reproductive health related to pubertal development, pregnancy and knowledge and perception of sexually transmitted diseases and acquired immune deficiency syndrome. The test contained 26 and 27 questions for girls in primary and middle schools, respectively. There were three research assistants in each room with 25 50 computers. One of these assistants issued unique codes and explained the background and objective of the survey, while the other two helped the participants log into the computers using the codes and provided guidance for completing the interview. The research staff uploaded and saved the data to the computer station at the end of the survey. The research staff did not interpret survey questions or observe the participants respond to specific survey questions.

We also conducted a physical examination and FFI for the girls who had completed CASI $(\mathrm{n}=3478)$. During FFI, the girls were also asked the same two main questions regarding their menarche. The trained clinicians first explained about menstrual periods to the girls and clarified that menarche was the first menstrual bleeding. The clinicians also asked necessary questions to help the participants recall their age at menarche (eg, 'what grade in school were you?' and 'what season was it?'). Girls were additionally examined for their pubertal development (eg, inspection and palpation of breast), and their medical and surgical histories were obtained.

\section{Statistics}

FFI, conducted by the clinicians, was used as the reference for determining the age at menarche. The capacity of CASI to correctly classify the status of menarche (dichotomous data: yes/no) was verified by tools of standard epidemiological indices, including sensitivity, specificity, predictive values and accuracy. ${ }^{15}$ There were three options for the CASI method: yes, no and unclear. Sensitivity was defined as the proportion of girls truly having menarche who were correctly grouped as such by the CASI. Specificity represented the proportion of girls who truly had no menarche and were correctly grouped as such by CASI. The predictive values were calculated as positive predictive value (PPV) and negative predictive value (NPV). The PPV was the proportion of girls truly having menarche among girls categorised as such by CASI. The NPV was the proportion of girls who truly had no menarche among girls categorised as such by CASI. The accuracy rate was the proportion of agreement between the classification of menarche status between CASI and FFI.

Subgroup analysis was conducted according to location (urban and rural), grades (grades 5-6: primary school, grades 7-9: junior middle school and grades 10-12: senior middle school), awareness in reproductive health and Tanner stages of breast development. The awareness of reproductive health was categorised into three groups according to the proportion of questions that the girls answered correctly $(<25 \%, 25 \%-50 \%$ and $>50 \%$ ); all the questions were assumed to be equally important for evaluating their awareness. Breast development was categorised as Tanner stages B1-B5, where stage 1 (B1) represented immaturity and stage 5 (B5) represented full maturity. ${ }^{16-18}$

A retrospective method was used to estimate the average age at menarche, and the means and percentiles (P5, P25, P50, P75 and P95) were calculated. Both Pearson and Spearman correlations were computed for determining the consistency of the age at menarche by 
CASI and FFI. The correlations between the difference in the age at menarche (CASI-FFI) and time interval between investigation and menarcheal onset were also calculated. Correlations were classified according to Cohen's criteria whereby a correlation of $0.10,0.30$ and 0.50 indicated a small, medium and large effect size, respectively. A Bland-Altman plot was constructed to measure the agreement of the age at menarche between FFI and CASI. ${ }^{19}{ }^{20}$ The age at investigation was computed according to the girls' birthdate, as obtained by FFI, and the date of investigation.

\section{RESULTS}

The mean age of the study participants was 14.3 years (SD: 2.46 years). Of the 3478 girls, $53.5 \%$ were from urban areas, $68.7 \%$ had a low awareness of reproductive health ( $<50 \%$ of correctly answered questions) and $24.1 \%$ answered $<25 \%$ of the questions correctly. Almost $60 \%$ of the participants were categorised in the late Tanner breast stages (B1: 6.4\%; B2: 10.5\%; B3: $23.5 \%$; B4: $28.1 \%$; B5: $31.5 \%$; table 1).

After physical examination, 2538 (73.0\%) and 2496 $(71.2 \%)$ girls who underwent FFI and CASI, respectively, were found to have attained menarche. Two hundred and three $(5.84 \%)$ girls were unclear about their status of menarche during CASI (table 1). Compared with FFI, the sensitivity, specificity, PPV, NPV and accuracy of CASI method were 0.97, 0.80, 0.99, 0.96 and 0.92 , respectively. The PPVs increased, while the NPVs decreased, with increasing grade and later Tanner breast stages. Among girls in grade 5, the sensitivity, specificity, PPV and accuracy were $<0.8$. The specificity was lower among girls from rural area (specificity: 0.71 ) or those with a lower awareness of reproductive health (specificity: 0.71 ; table 1 ).

Among the 2458 girls who reported their menarcheal age, the average age at menarche was 12.5 years in both FFI and CASI. The Pearson and Spearman correlation coefficients for the age at menarche between CASI and FFI was 0.802 and 0.869 , respectively. The partial correlation coefficient was 0.728 after adjusting for locations, grades and awareness of reproductive health. The Pearson, Spearman and partial correlation coefficients were increased with increasing grade and awareness in reproductive health. Among girls in grade 5, the Pearson, Spearman and partial correlation coefficients were $0.369,0.678$ and 0.335 , respectively (table 2). The mean difference in the age at menarche between CASI and FFI was 0.05 years (SD: 0.82 years). Approximately $>95 \%$ and $76.3 \%$ of the difference in the age at menarche between CASI and FFI ranged within 12 months and 3 months, respectively (figure 1). The time interval between the age at investigation and age at menarche had a small effect on this difference (correlation: 0.189) in the investigated population (figure 2).

\section{DISCUSSION}

In the present study, we examined the capacity of CASI to correctly classify the status of menarche and validate the self-reported age at menarche compared with FFI, the reference method. We generally found that the validity of the status of menarche obtained by CASI was satisfactory due to higher scores obtained for the indices (ie, sensitivity, specificity, predictive values and accuracy). PPVs increased and NPVs decreased with increasing grades and Tanner breast stages. This negative correlation of PPV and NPV was probably attributed to the increased prevalence of menarche across the age groups. ${ }^{15}$ The Tanner stages of breast development and school grades generally increased with age. ${ }^{21} 22$

We also found that the menarcheal age reported in CASI closely agreed with that in FFI. The discrepancy in the age at menarche between these two interviews varied by an average of 0.6 month. The average age at menarche in CASI was 12.5 years, which was similar to that of FFI. Dorn et $a l^{23}$ conducted a cross-sequential study in 253 girls categorised into four age cohorts to explore the reliability of age at menarche across time points and methods. They reported large discrepancies in the age at menarche between the phone interview and FFI across 12 time points.

Moreover, we found lower scores of sensitivity, specificity, PPV and accuracy and a medium correlation of the age at menarche between CASI and FFI among girls in grade 5 . We also found a relatively low specificity in those living in rural areas and those with a low awareness in reproductive health. Such lower scores would be due to the low awareness in reproductive health. ${ }^{24}$ In our study, girls in grade 5 attended primary school and were aged 10.9 years on average. In China, most girls initially become informed regarding reproductive health, including menarche, in grade 5. ${ }^{25}$ Girls who were not knowledgeable about menarche would probably not fully understand the questions that were asked from them during the interviews, which can result in inaccurate answers. ${ }^{26}$ In our study, 603 (17.3\%) girls did not know what menstruation was, and girls in grade 6 or lower had lower knowledge of menstruation than girls in grade 7 or higher. According to the subgroup analysis regarding the girls' knowledge of reproductive health, including menstruation, the correlation in the age at menarche between CASI and FFI was poorer among girls with low knowledge. Girls living in rural areas of China may have less access to computers and poorer computer skills compared with those in urban areas, which could impact the ability of the girls from rural areas to respond accurately during the CASI. Furthermore, the accuracy of the timing of menarche can be influenced by recall bias from the study participants. ${ }^{527}$ In our study, the time from menarche to study participation had only a small effect on the difference between FFI and CASI. The correlations in the age at menarche between interview methods also showed similar results across time. ${ }^{26}$ 


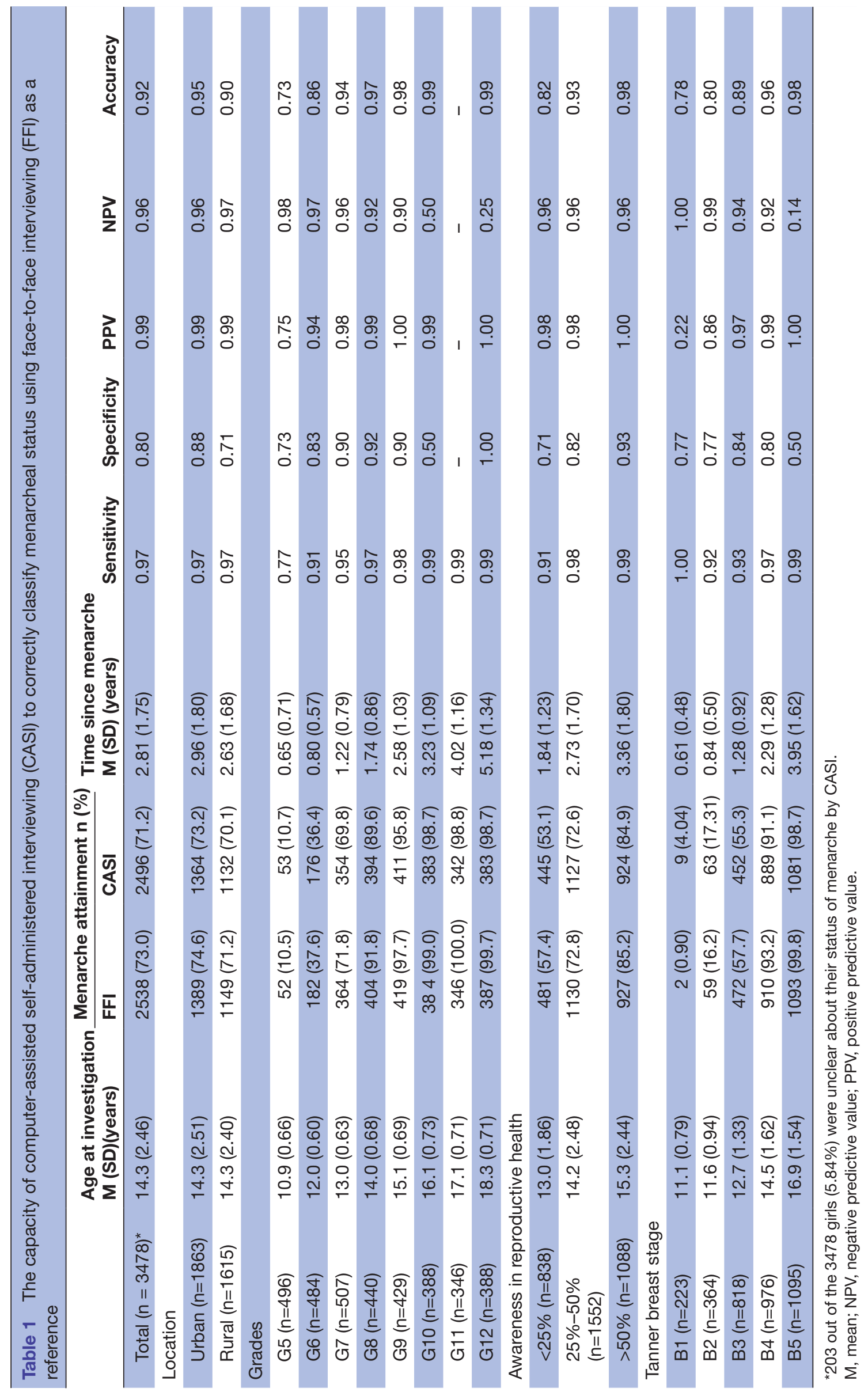




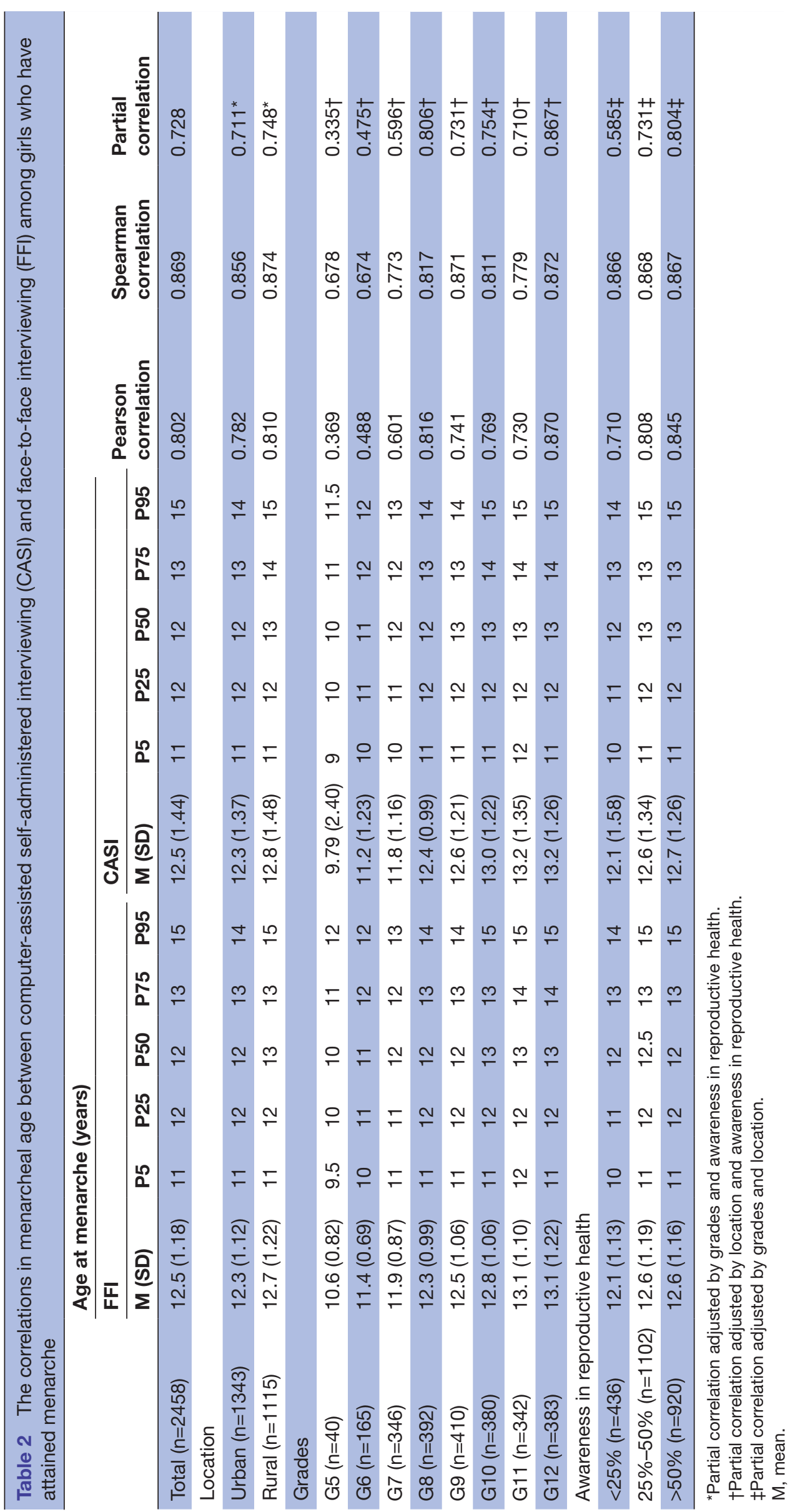




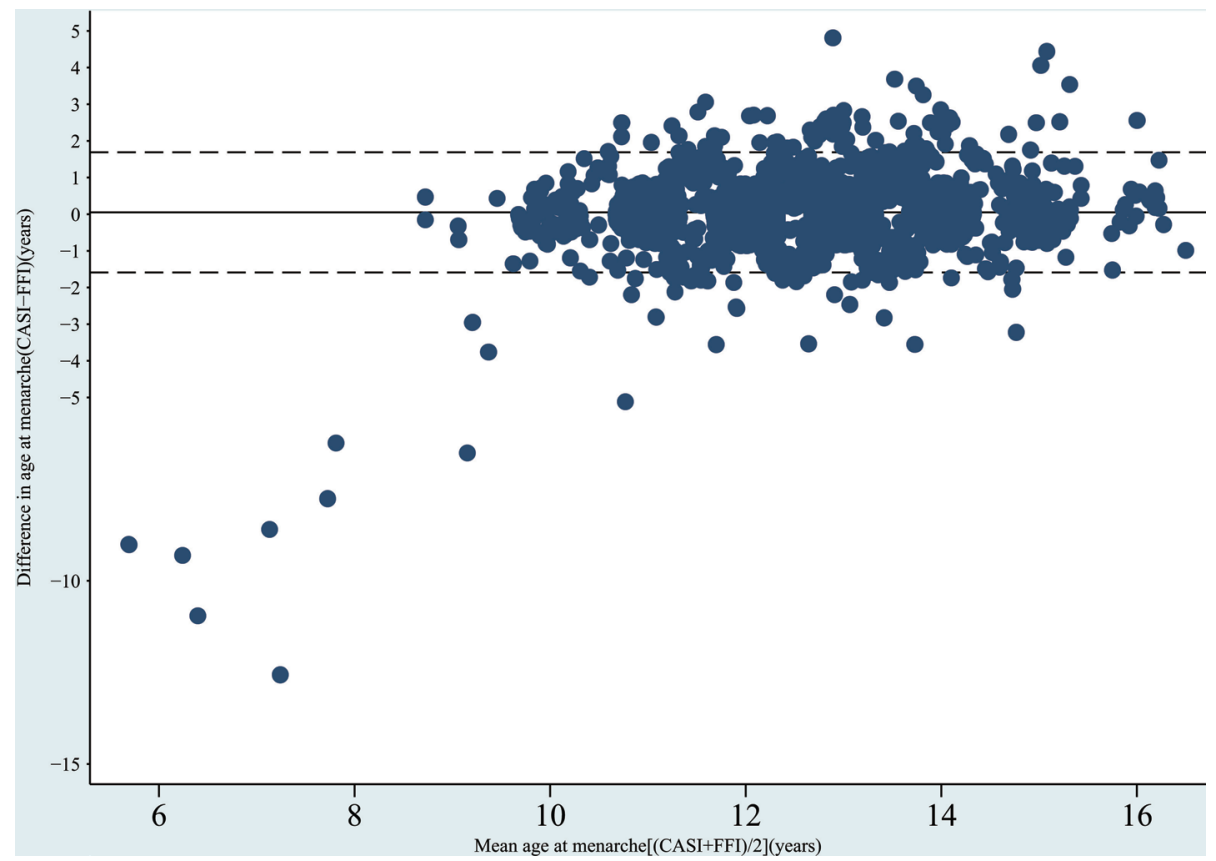

Figure 1 Bland-Altman plot showing agreement of age at menarche between CASI and FFI.The line in the middle represents the mean difference in age at menarche between the two instruments $(M=0.05)$, and the two other dotted lines represent the upper and lower limits of agreement $(\mathrm{M} \pm 2 \mathrm{SD}=-1.59,1.69)$. CASI, computer-assisted self-administered interview; FFI, face-toface interview.

In the present study, conducted in a large population of 3478 schoolgirls in six provinces of China nationwide, we validated the use of CASI for determining girls' status and age of menarche attainment using FFI as a reference. FFI has been considered a valid and reliable method ${ }^{26}$ for measuring menarcheal age based on long-term ${ }^{28-31}$ and short-term recalls. ${ }^{52}{ }^{33}$ However, our study was based on a cross-sectional study, and the data regarding menarche collected using both CASI and FFI were self-reported and not prospectively recorded.

\section{CONCLUSIONS}

The timing of menarche investigated in CASI was valid among all Chinese schoolgirls, except for those in grade 5 or lower, which suggested that the CASI method should

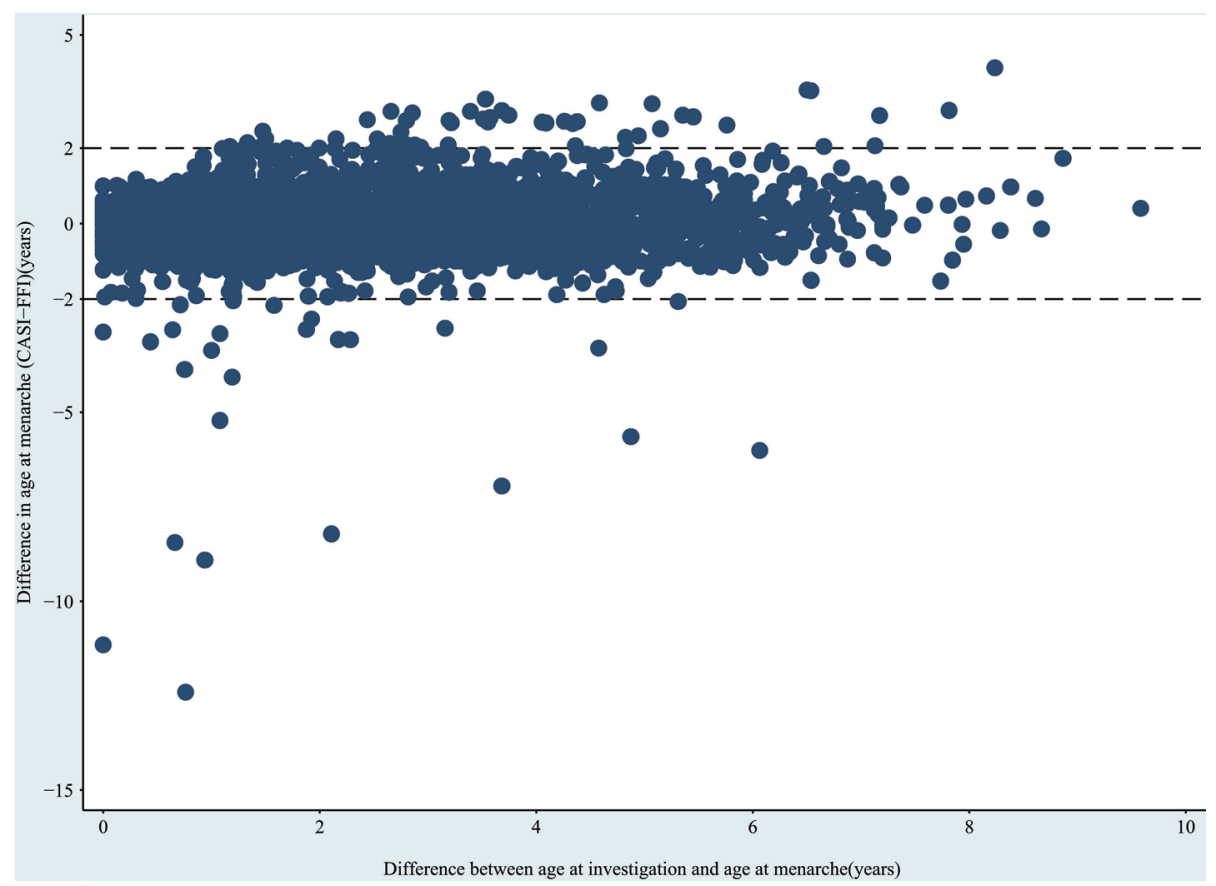

Figure 2 Correlation between differences CASI and FFI across the time interval since menarche to investigation (partial correlation=0.189). $\mathrm{CASI}$, computer- assisted self-administered interview; FFI, face-to-face interview. 
be used with caution among Chinese primary school students.

Acknowledgements We would like to thank the administrators of field education bureau and teachers of the field schools for their assistance with facilities and arranging the computer-assisted self-administered interviews and physical examinations for this study. We also thank Editage (http://www.editage.cn) for English language editing.

Contributors WZ, CL, SZ and SL conceptualised and designed the study; YM, $\mathrm{YZ}$ and $\mathrm{XZ}$ collected and cleaned the data; $\mathrm{YM}, \mathrm{QL}$ and $\mathrm{XZ}$ conducted statistical analysis and drafted the manuscript; XT, SL, YZ and QL interpreted the results; CL, WZ and SZ contributed to discussion; and all authors reviewed and approved the final manuscript.

Funding This study was funded by the National Key Technology Research and Development Program of China (Grant No. 2012BAl32B02) and the National Science and Technology Infrastructure Program of China (Grant No. 2013FY110500).

Competing interests None declared.

Patient consent Parental/guardian consent obtained.

Ethics approval The study was reviewed and approved by the institutional review board of Shanghai Institute of Planned Parenthood Research (2012-01).

Provenance and peer review Not commissioned; externally peer reviewed.

Data sharing statement The authors are willing to share any of the data collected during validation process that are not in this published manuscript or the online supplementary material.

Open Access This is an Open Access article distributed in accordance with the Creative Commons Attribution Non Commercial (CC BY-NC 4.0) license, which permits others to distribute, remix, adapt, build upon this work non-commercially, and license their derivative works on different terms, provided the original work is properly cited and the use is non-commercial. See: http://creativecommons.org/ licenses/by-nc/4.0/

C Article author(s) (or their employer(s) unless otherwise stated in the text of the article) 2018. All rights reserved. No commercial use is permitted unless otherwise expressly granted.

\section{REFERENCES}

1. Tondo L, Pinna M, Serra G, et al. Age at menarche predicts age at onset of major affective and anxiety disorders. Eur Psychiatry 2017;39:80-5.

2. Canoy D, Beral V, Balkwill A, et al. Age at menarche and risks of coronary heart and other vascular diseases in a large UK cohort. Circulation 2015;131:237-44.

3. Yang L, Li L, Millwood IY, et al. Age at menarche and risk of major cardiovascular diseases: Evidence of birth cohort effects from a prospective study of 300,000 Chinese women. Int J Cardiol 2017;227:497-502.

4. Hediger ML, Stine RA. Age at menarche based on recall information. Ann Hum Biol 1987;14:133-42.

5. Koo MM, Rohan TE. Accuracy of short-term recall of age at menarche. Ann Hum Biol 1997;24:61-4.

6. Uskul AK. Women's menarche stories from a multicultural sample. Soc Sci Med 2004;59:667-79.

7. Chang YT, Hayter M, Wu SC. A systematic review and metaethnography of the qualitative literature: experiences of the menarche. J Clin Nurs 2010;19:447-60.

8. Turner CF, Ku L, Rogers SM, et al. Adolescent sexual behavior, drug use, and violence: increased reporting with computer survey technology. Science 1998;280:867-73.
9. Rew L, Horner SD, Riesch L, et al. Computer-assisted survey interviewing of school-age children. ANS Adv Nurs Sci 2004;27:129-37.

10. Le LC, Blum RW, Magnani R, et al. A pilot of audio computerassisted self-interview for youth reproductive health research in Vietnam. J Adolesc Health 2006;38:740-7.

11. Watson PD, Denny SJ, Adair V, et al. Adolescents' perceptions of a health survey using multimedia computer-assisted self-administered interview. Aust N Z J Public Health 2001;25:520-4.

12. Newman JC, Des Jarlais DC, Turner CF, et al. The differential effects of face-to-face and computer interview modes. Am J Public Health 2002;92:294-7.

13. Cristóbal-Narváez $P$, Sheinbaum T, Ballespí S, et al. Impact of Adverse Childhood Experiences on Psychotic-Like Symptoms and Stress Reactivity in Daily Life in Nonclinical Young Adults. PLoS One 2016;11:e0153557.

14. Lian Q, Zuo X, Mao Y, et al. Anorexia nervosa, depression and suicidal thoughts among Chinese adolescents: a national schoolbased cross-sectional study. Environ Health Prev Med 2017;22:30.

15. Hajian-Tilaki K. Receiver Operating Characteristic (ROC) Curve Analysis for Medical Diagnostic Test Evaluation. Caspian J Intern Med 2013;4:627-35.

16. Marshall WA, Tanner JM. Variations in pattern of pubertal changes in girls. Arch Dis Child 1969;44:291-303.

17. Bordini B, Rosenfield RL. Normal pubertal development: part II: clinical aspects of puberty. Pediatr Rev 2011;32:281-92.

18. Chipkevitch $\mathrm{E}$. [Clinical assessment of sexual maturation in adolescents]. J Pediatr 2001;77(Suppl 2):135-42.

19. Bland JM, Altman DG. Measuring agreement in method comparison studies. Stat Methods Med Res 1999;8:135-60.

20. Bland JM, Altman DG. Statistical methods for assessing agreement between two methods of clinical measurement. Lancet 1986;1:307-10.

21. Sun Y, Tao FB, Su PY, Py S, et al. National estimates of the pubertal milestones among urban and rural Chinese girls. J Adolesc Health 2012;51:279-84.

22. Anderson SE, Must $A$. Interpreting the continued decline in the average age at menarche: results from two nationally representative surveys of U.S. girls studied 10 years apart. J Pediatr 2005;147:753-60.

23. Dorn LD, Sontag-Padilla LM, Pabst S, et al. Longitudinal reliability of self-reported age at menarche in adolescent girls: Variability across time and setting. Dev Psychol 2013;49:1187-93.

24. Yu J. Teenage sexual attitudes and behaviour in China: a literature review. Health Soc Care Community 2012;20:561-82.

25. Hong J, Fongkaew W, Senaratana W, et al. Development of a theory-based sexual and reproductive health promotion and HIV prevention program for Chinese early adolescents. Nurs Health Sci 2010;12:360-8.

26. Dorn LD, Sontag-Padilla LM, Pabst S, et al. Longitudinal reliability of self-reported age at menarche in adolescent girls: variability across time and setting. Dev Psychol 2013;49:1187-93.

27. Castilho SD, Nucci LB, Assuino SR, et al. [The importance of memory bias in obtaining age of menarche by recall method in Brazilian adolescents]. Arq Bras Endocrinol Metabol 2014;58:394-7.

28. Livson $\mathrm{N}, \mathrm{McNEILL} \mathrm{D,} \mathrm{Nd} \mathrm{M}$. The accuracy of recalled age of menarche. Hum Biol 1962;34:218-21.

29. Damon A, Bajema CJ. Age at menarche: Accuracy of recall after thirty-nine years. Hum Biol 1974;46:381-4.

30. Casey VA, Dwyer JT, Coleman KA, et al. Accuracy of recall by middle-aged participants in a longitudinal study of their body size and indices of maturation earlier in life. Ann Hum Biol 1991;18:155-66.

31. Must A, Phillips SM, Naumova EN, et al. Recall of early menstrual history and menarcheal body size: after 30 years. how well do women remember? Am J Epidemiol 2002;155:672-9.

32. Bergsten-Brucefors $A$. A note on the accuracy of recalled age at menarche. Ann Hum Biol 1976;3:71-3.

33. Koprowski C, Coates RJ, Bernstein L. Ability of young women to recall past body size and age at menarche. Obes Res 2001;9:478-85. 